\title{
Archéologie participative avec les Wayana dans les Tumuc-Humac (Mont Mitaraka, crique Alama, Guyane Française)
}

Marie Fleury, Aimawale Opoya, Aitalewa Palanaiwa, Anaima Putpu, Daniel Sabatier et Martijn van den Bel

\section{(2) OpenEdition}

Journals

Édition électronique

URL : https://journals.openedition.org/ethnoecologie/6750

DOI : $10.4000 /$ ethnoecologie.6750

ISSN : 2267-2419

Éditeur

Laboratoire Éco-anthropologie

Référence électronique

Marie Fleury, Aimawale Opoya, Aitalewa Palanaiwa, Anaima Putpu, Daniel Sabatier et Martijn van den Bel, «Archéologie participative avec les Wayana dans les Tumuc-Humac (Mont Mitaraka, crique Alama, Guyane Française) », Revue d'ethnoécologie [En ligne], 19 | 2021, mis en ligne le 30 juin 2021, consulté le 18 décembre 2021. URL : http://journals.openedition.org/ethnoecologie/6750 ; DOI : https://doi.org/10.4000/ethnoecologie.6750

Ce document a été généré automatiquement le 18 décembre 2021.

\section{cc) (†)}

Revue d'ethnoécologie est mis à disposition selon les termes de la licence Creative Commons Attribution - Pas d'Utilisation Commerciale - Pas de Modification 4.0 International. 


\title{
Archéologie participative avec les Wayana dans les Tumuc-Humac (Mont Mitaraka, crique Alama, Guyane Française)
}

\author{
Marie Fleury, Aimawale Opoya, Aitalewa Palanaiwa, Anaima Putpu, Daniel \\ Sabatier et Martijn van den Bel
}

\section{Introduction}

1 La connaissance scientifique du sud de la Guyane reste très parcellaire : un tiers de la Guyane est inexploré en raison de nombreuses zones inaccessibles en particulier dans le grand Sud forestier. Ce constat vrai pour l'inventaire des espèces biologiques l'est encore plus en ce qui concerne l'archéologie. Les quelques missions archéologiques précédant celle-ci dans la région des Tumuc-Humac avaient toutefois permis d'identifier quelques sites à polissoirs et objets erratiques (poteries, haches en pierre) ou encore le site « couronné » de Yaou (Abonnenc 1952, Petitjean-Roget 1991, Mestre et al. 2013). Sur le site de la Borne 1 (frontière entre la France et le Brésil), a été découvert un ensemble de géoglyphes ou alignements qui se trouvent sur le territoire brésilien (Hurault et al. 1963). En 2006, une prospection archéologique a mis au jour plusieurs abris sous-roche dans les alentours de ce même site de la Borne 1 (Migeon 2008). D'un statut précieux sont également les peintures rupestres du Mamilipan et la roche gravée du Marouini (Mazière \& Mazière 1997). Mais, comme nous pouvons constater, il s'agit principalement de sites spectaculaires et peu de sites d'habitat (Duin 2004, 2009). Pourtant, l'habitat est omniprésent dans la forêt guyanaise et lors des prospections pédestres, les archéologues trouvent en moyenne un site par kilomètre carré.

2 Lors de la mission naturaliste «Planète revisitée " en mars 2015 organisée par le Muséum national d'Histoire naturelle et l'ONG Pro-Natura international dans la région du Mitaraka $^{1}$ (Figure 1), différents lieux de prospection ont également livré des 
éléments archéologiques, notamment des fragments de céramique et un site fossoyé ou " montagne couronnée ». Ces découvertes méritaient une deuxième mission, mais cette fois en sciences humaines, dans une perspective ethno-historique et archéologique. Dans un premier temps, il s'agissait de réaliser une prospection pédestre de la zone afin de mieux comprendre la relation diachronique entre l'homme et son environnement. Elle a été effectuée par une équipe constituée d'un archéologue de l'INRAP, d'une ethnobotaniste du MNHN, d'un écologue/botaniste de l'IRD et de trois membres de la communauté Wayana, tous co-auteurs de cette publication.

Figure 1 : Carte de la Guyane avec la situation de la région du Mitaraka prospectée

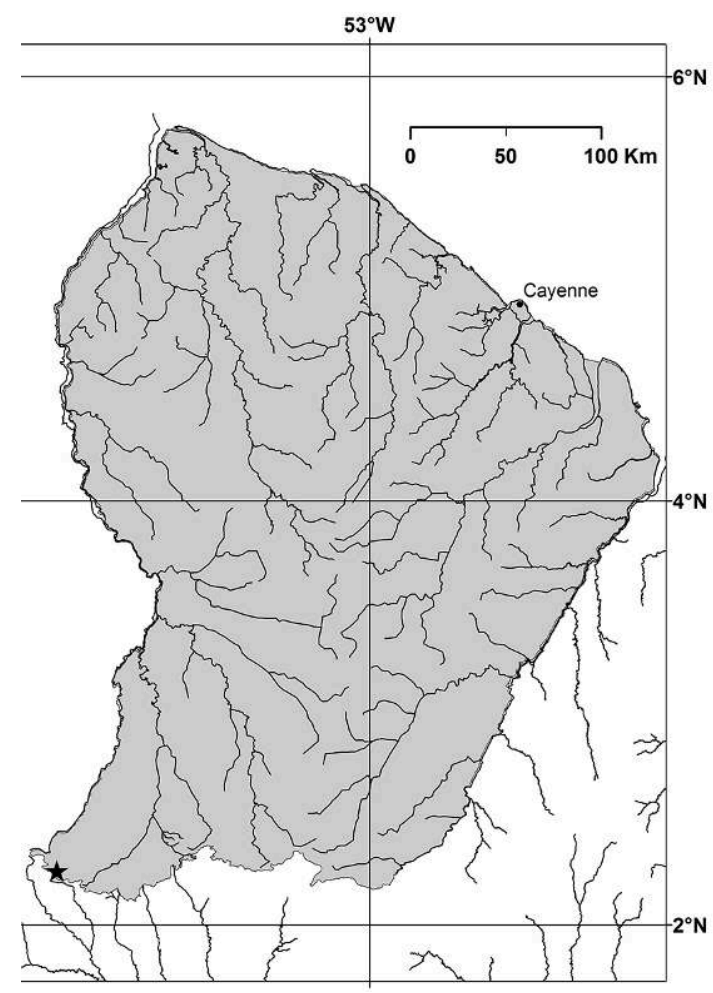

\section{De la cartographie participative à l'archéologie participative}

Cette mission archéologique se trouve aussi dans la continuité d'une démarche de recherche participative menée par Marie Fleury avec les Wayana, à propos des anciens sites habités sur le haut Maroni, en partenariat avec le Parc Amazonien de Guyane. En effet, les Wayana sont très intéressés à mieux connaître leur histoire et celle de leur arrivée en Guyane, comme l'a montré le succès de ces différentes missions de cartographie de leurs anciens villages sur le Marouini en 1999, puis sur le haut Litani en 2013 et 2015 (Fleury et al. 2016, Fleury 2018).

L'archéologie nous permet de remonter un peu plus loin dans le temps car selon les sources orales, la zone de prospection correspond à un ancien lieu de vie des Wayana durant leur longue migration du Brésil vers la Guyane. En effet, la genèse de leur peuple se déroule dans les Tumuc-Humac (Chapuis 2007). Elle résulte de la fusion des différents groupes culturels (clans) qui a mené à la création du groupe Wayana vers le XVIII ${ }^{e}$ siècle. 
Ces différents groupes vivaient sur le Jari ou le Paru au Brésil depuis au moins le $\mathrm{xv}^{\mathrm{e}}$ siècle, jusqu'à ce qu'ils en soient chassés par des traqueurs d'esclaves armés par les traiteurs portugais (Grenand 1982).

Ils se sont alors réfugiés dans la région des Tumuc-Humac, où ils ont vécu de nombreuses années avant leur longue descente du Maroni par deux de ses principaux affluents : le Marouini et la Litani. Les précédents travaux de cartographie participative avec les Wayana ont permis de retracer cette descente des affluents du Maroni, notamment le long de la Litani grâce à la mémoire des Anciens qui nous ont montré l'emplacement des lieux de vie ancestraux. Plusieurs missions (en août 2013 et août 2015) ont été réalisées en pirogue avec des anciens Wayana, porteurs de savoirs, et des jeunes qui souhaitaient se réapproprier leur histoire. Si, de mémoire, les Anciens nous avaient cité une cinquantaine d'anciens villages, ces missions de reconnaissance nous ont permis d'identifier environ 130 toponymes, dont 70 anciens villages sur la Litani (Fleury et al. 2016).

Les plus anciens villages localisés se situent au pied des Tumuc-Humac, certains sur la haute Litani et les autres au pied du Mitararaka (Fleury 2018), et notamment le long de la crique Alama :

«les gens se sont croisés aux Tumuc-Humac comme à Alama patatpë («ancien village Alama») où les gens du Jari et de tous côtés venaient s'installer. C'est l'un des premiers villages du côté français de la ligne du partage des eaux »" (Pidima 2015 in Fleury et al. 2016).

7 Selon Chapuis (2007) les Alamajana (littéralement "les gens d'Alama ») sont un des groupes fondateurs des Tiiliyo, qui étaient alliés avec les Kukujana (un des clans Wayana), et combattaient avec eux. Mais on ne sait pas s'il y a un lien avec la crique Alama. En effet «alama » en wayana signifie mouche mélipone (ou mouche à miel), donc alamajana signifie également les "gens de l'abeille». Ce nom fait référence à un animal, comme beaucoup d'autres clans totémiques (les gens des singes hurleurs, des lucioles, etc..). On peut envisager deux hypothèses : soit des Alamajana occupaient cette crique autrefois et ils lui ont laissé leur nom (ou l'inverse). Soit la crique fut envahie par des Mélipones à un moment donné ${ }^{2}$, et elle en porte le nom car le miel est une denrée recherchée, comme beaucoup d'autres lieux dont les toponymes se raccrochent à des anecdotes, des mythes, ou des espèces animales ou végétales devenant des marqueurs de paysages (Fleury et al. 2016).

8 Quoi qu'il en soit, les Wayana y ont vécu à un moment donné, comme le confirment les témoignages historiques. Par exemple, le lieutenant Vidal, envoyé pour reconnaître la frontière franco-hollandaise en 1861, note la présence d'un village à l'embouchure de la crique Alama, village qui faisait le lien avec les villages du Jari, et qui facilitait les relations de commerce avec les Boni (Vidal 2012). L'explorateur Coudreau relève quant à lui, trente ans plus tard, en 1890 (Figure 2), les traces d'un ancien village plus en amont sur la crique Alama à deux jours de marche de l'embouchure (Coudreau 1893). On peut en conclure, d'après ces témoignages que la crique Alama fut un des lieux de vie des différents groupes proto-Wayana. De plus les Kukujana (ou «vrais Wayana ») comme les appellent parfois les Wayana actuels, qui auraient vécu sur cette crique, sont localisés un peu plus tard (fin $\mathrm{XIX}^{\mathrm{e}}$ ) sur la crique Sinale, sur le haut Marouini (qui n'est pas très loin à vol d'oiseau, (ou plutôt à enjambées d'Amérindiens). C'est à partir de là que le clan de Twenké l'Ancien a commencé sa migration jusqu'à l'actuel village de Twenké sur la Litani (Fleury 2018). Selon les différents témoignages historiques, ces 
ancêtres des Wayana ont vécu dans cette région des Tumuc-Humac pendant au moins cent ans (1766-1862).

Figure 2 : Extrait de la carte de Coudreau (1891) avec la position des Wayana notamment dans la région de la crique Alama

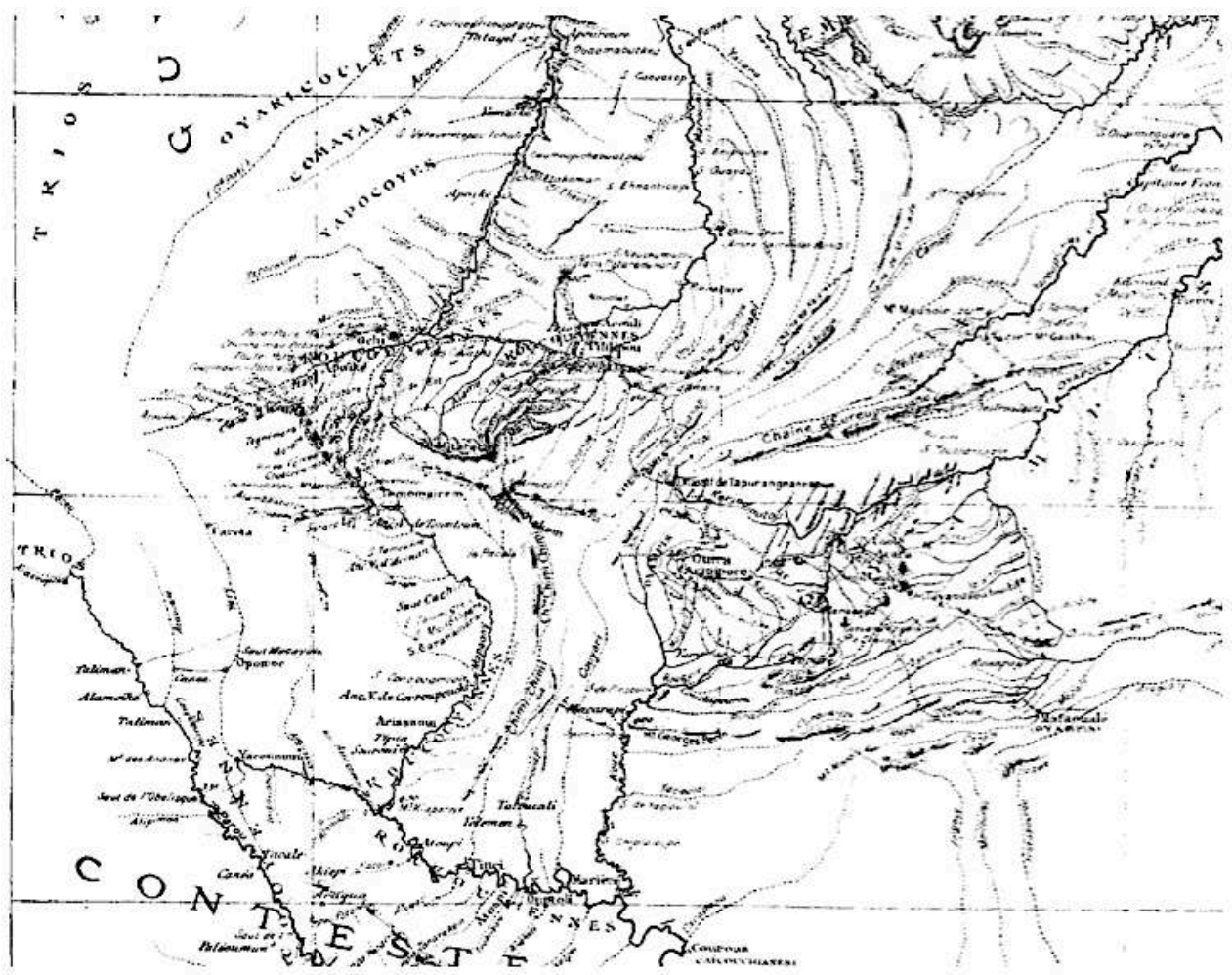

\section{Le projet d'archéologie participative : genèse et méthodologie}

C'est pour toutes ces raisons que, lors de la mission «Planète revisitée ", qui s'est déroulée en 2015, près de la source de la crique Alama, le présent projet d'archéologie participative avec les Wayana nous est venu naturellement à l'esprit. D'autant plus que la croûte noire d'un tesson que nous avons trouvé accidentellement sur une des collines à proximité de la crique, a été datée, avec une forte probabilité, du début du xix siècle. Cette période coïncide justement avec la période d'occupation des Tumuc-Humac par les proto-Wayana dans cette région...

10 Les Wayana étant toujours très enthousiastes à l'idée de mieux connaître leur propre histoire et territoire, il n'a pas été difficile de trouver plusieurs personnes motivées pour nous accompagner sur le terrain :

- Anaima Putpu, dit « Mimi Siku» : arrivé enfant sur la Litani, il est installé depuis plus de cinquante ans à Antécume pata, et a fait de nombreuses fois le chemin entre le Jari et la Litani, avec son père et son oncle. Il a accepté de nous accompagner car il connaît bien la région. Il a aussi participé à plusieurs missions avec des scientifiques, notamment le botaniste Jean-Jacques de Granville dans les années 1970 ;

- Aitalewa Palanaiwa, dit Capitaine Barbosa, chef coutumier du village d'Antécume pata, n'avait jamais voyagé auparavant dans les Tumuc-Humac, mais il connaît bien 
l'histoire et les traditions de son peuple ;

- Aimawale Opoya : jeune Wayana, descendant du chef Opoya, fondateur du village Taluwen, et qui s'intéresse énormément à sa culture. Artiste reconnu, il a contribué aux recherches de Jean Chapuis comme traducteur lors de ses travaux sur le terrain. Il a également participé aux travaux de cartographie participative, notamment par la traduction des témoignages enregistrés des Anciens. Il a été récemment nommé chef du village de Taluwen.

$11 \mathrm{Du}$ point de vue archéologique, le premier projet prévoyait d'emmener trois archéologues, mais pour des raisons de financement et d'organisation, l'équipe s'est réduite à un seul : Martijn van den Bel, archéologue à l'INRAP de Cayenne depuis 2004. Deux autres chercheurs participaient à la mission :

- Daniel Sabatier, écologue et botaniste spécialiste de la forêt guyanaise qu'il parcourt depuis une quarantaine d'années.

- Marie Fleury, ethnobotaniste travaillant depuis plus de vingt-cinq ans avec les Wayana et ayant réalisé des travaux de cartographie sur leurs territoires depuis 1999.

Une partie de l'équipe a été déposée en hélicoptère sur une savane roche à environ trois kilomètres de l'ancien camp de la mission «Planète revisitée ». À l'aide de machettes et d'une tronçonneuse, l'ancienne $\mathrm{DZ}$ a été dégagée pour la dépose de la deuxième rotation d'hélicoptère qui amenait le reste de l'équipe, le matériel de la mission, et les vivres pour deux semaines, entre le 8 et le 20 octobre 2018 (Figure 3).

Figure 3 : L'équipe avec le pilote de l'hélicoptère sur la DZ

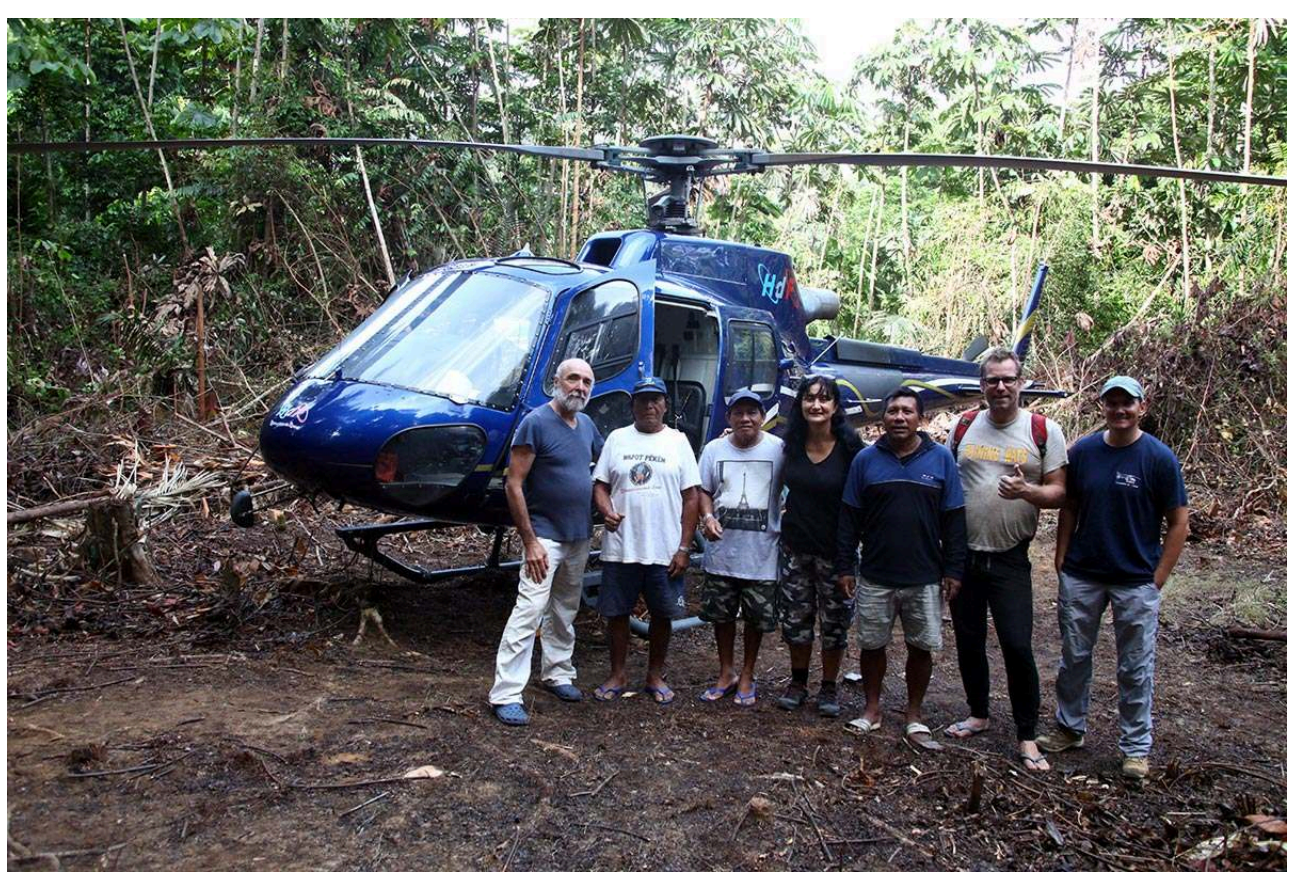

Cliché D. Sabatier

\section{Objectifs de cette prospection pédestre}

Le premier objectif était de visiter les abords de la crique Alama et les collines proches, propices à l'installation de lieux de vie; les voies de circulation (layons) et les placettes botaniques établies lors de la mission «Planète revisitée » servant de points d'appui 
aux prospections. Il s'agissait de trouver des indices archéologiques nouveaux mais aussi de revisiter les sites archéologiques déjà découverts afin de consolider les données scientifiques existantes. Aucune fouille par creusement n'était autorisée.

Le deuxième objectif, si le temps le permettait, était de visiter des lieux plus éloignés dans la direction du mont Mitaraka, mais toujours en bordure de la crique Alama.

N'ayant pas obtenu le financement pour la réalisation d'un levé topographique LIDAR, c'est uniquement par prospection pédestre et évaluation visuelle des artefacts du relief que nous avons recherché les sites à fossés.

Quatre layons principaux (A-D) partant du camp de base parcourent la zone de recherche, formant une croix (Figure 4a). On marchait en groupe dispersé et on visait chaque jour d'étudier deux ou trois collines ou plateaux. Lors de nos marches, on inspectait les buttes de déracinement des arbres et les trous d'animaux afin de trouver du mobilier archéologique et on discutait du paysage, des espèces d'arbres et palmiers rencontrés afin d'évaluer l'aspect plus ou moins secondarisé de la forêt (présence et abondance d'espèces pionnières ou sciaphiles, de lianes, de palmiers Astrocarium sciophillum etc.). Un total de 80 kilomètres linéaires a été parcouru lors de cette mission soit huit kilomètres par jour en forêt tropicale avec un paysage très accidenté (Figure 4). Les indices ont été géo-référencés à l'aide d'un GPS ainsi que la trace de toutes nos prospections. Afin de visiter l'inselberg Tukusipan et le site fossoyé situé à proximité, nous avons également fait un bivouac de deux nuits pour ces sites les plus éloignés.

Figure 4a : Zones de relevés de la mission Mitaraka, lieu de la mission « Planète revisitée » en 2015, et de notre mission d'archéologie participative en 2018 : layons et traces principales de prospection

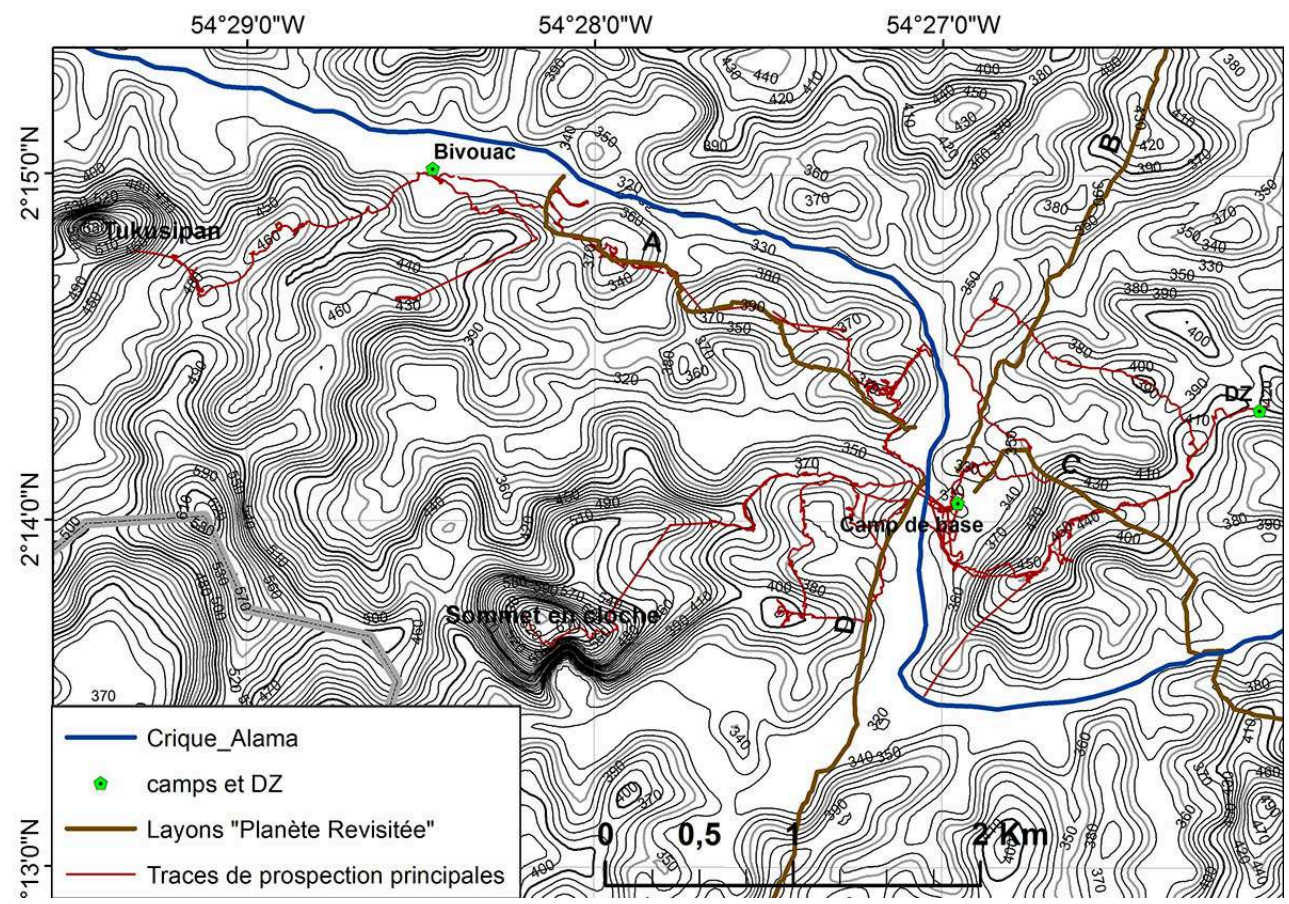


Figure 4b : Zones de relevés de la mission Mitaraka, lieu de la mission « Planète revisitée » en 2015, et de notre mission d'archéologie participative en 2018 : sites et indices archéologiques

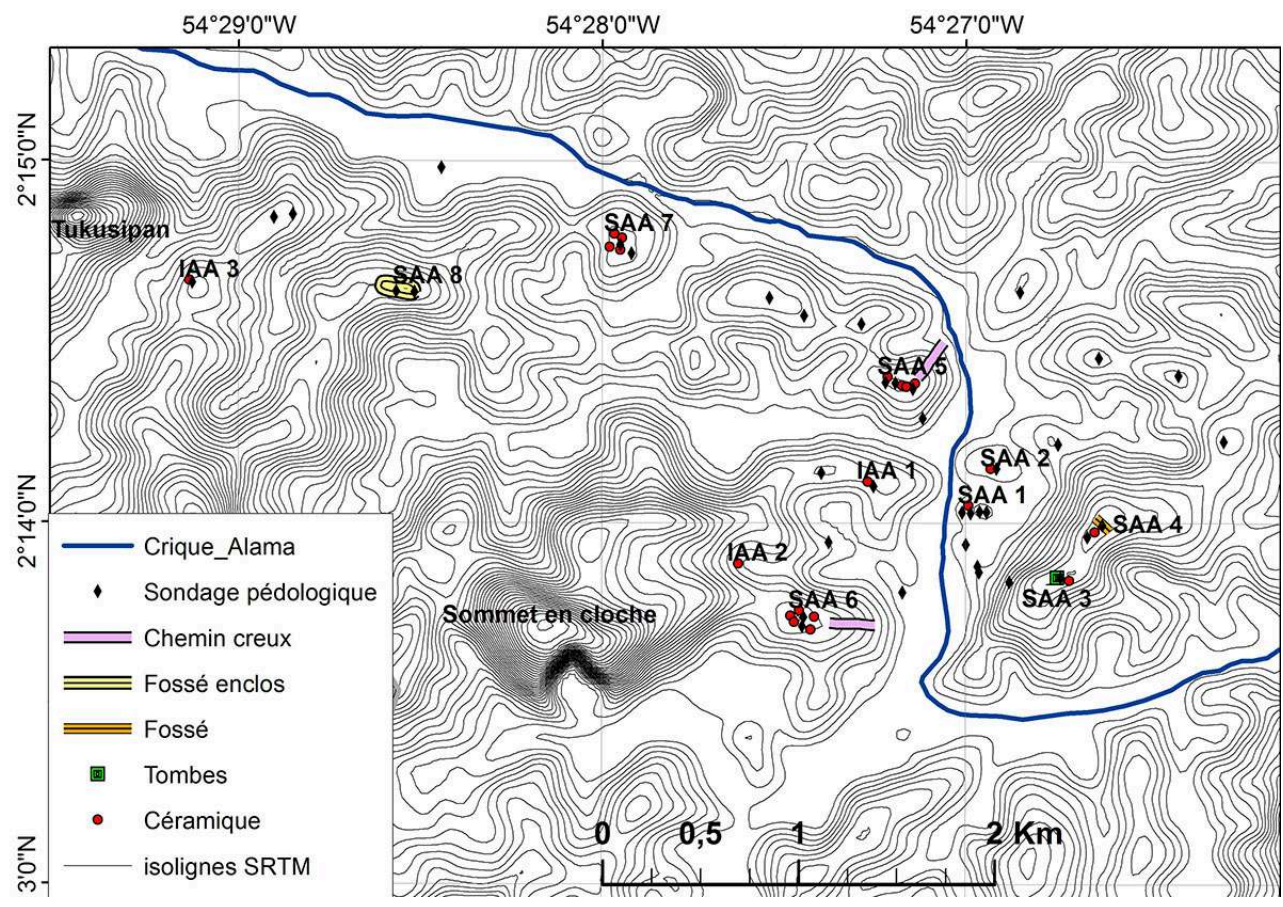

17 Sur les sommets et pentes nous avons réalisé des sondages pédologiques jusqu'à $110 \mathrm{~cm}$ de profondeur à l'aide d'une tarière du type dit «Edelman » à tête de sept centimètres de diamètre. La séquence pédologique a été décrite et photographiée sur place. Des charbons pour datations et des échantillons de sol sombre (100 grammes environ) ont été pris pour analyse.

\section{Résultats Archéologiques (Figure 4b)}

Cette prospection dans la région de la crique Alama (Figure 5) nous a révélé huit sites et trois indices archéologiques. Les sites archéologiques sont pour la plupart des sites en hauteur avec un assez large plateau compatible avec un habitat et ses activités annexes. 
Figure 5 : Crique Alama à proximité de la cascade

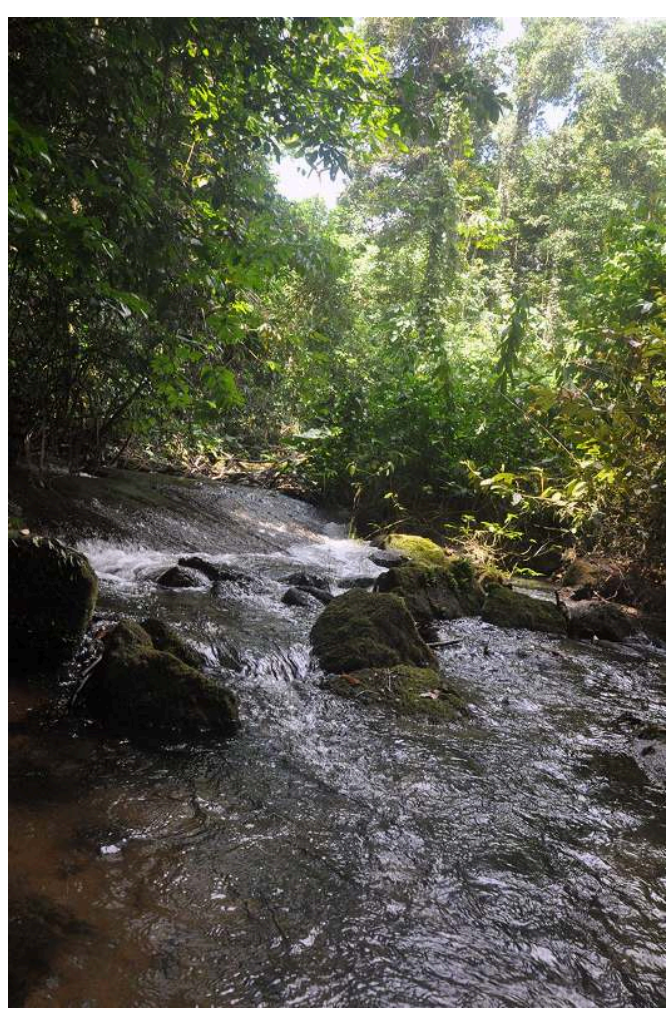

Cliché M. Fleury

\section{Les sites archéologiques Alama (SAA)}

19 - SAA 1 : Camp de base « Planète revisitée »

Ce site se trouve sur la petite colline où avait été établi le grand camp de base de la mission "Planète revisitée » ainsi que la DZ. Des fragments de céramique ont été trouvés en 2015 lors du creusement des toilettes sèches mais lors de notre visite aucun fragment n'a été trouvé malheureusement. Quatre sondages à la tarière ont montré une couche sombre et enfouie sur la pente Est.

- SAA 2 : Ancien camp de l'ONF

Ce site se trouve également sur une petite colline à sommet plat dans la plaine de la Crique Alama à environ 200 mètres vers le nord du site SAA 1. Deux tessons ainsi qu'un niveau sombre enfoui marquent ce site où se trouvait le camp de base de l'ONF en 2015.

- SAA 3 : Site funéraire?

Ce site se trouve sur la pointe méridionale d'un plateau élevé qui domine la Crique Alama. Une bonne quantité de céramique $(\mathrm{n}=30)$ a été trouvée en surface ainsi qu'un niveau bien sombre, enfoui. Selon les Wayana, il s'agit d'un site funéraire à plusieurs fosses rectangulaires.

- SAA 4 : Éperon barré

Ce même plateau a livré un autre site plus vers le nord après une cambrouse et un talweg. Le sommet très plat de ce plateau semblait avoir connu un « arasage ». Plus loin, nous avons identifié un fossé rectiligne de quatre mètres de large sur 30 mètres de long, qui barre le plateau mais n'atteint pas ses bords. Il a une profondeur de deux mètres environ ainsi que des petits bourrelets. Nous avons récolté 16 fragments de céramique dans des chablis. Par ailleurs, la croûte noire présente sur un petit fragment 
de céramique, rapporté par Marie Fleury lors de la mission 2015, provenait de ce site : il a été daté par AMS en livrant une date calibrée au début du XIXe siècle (Figure 6).

Figure 6 : Tableau des datations archéologiques

\begin{tabular}{|c|c|c|c|c|c|c|c|c|c|}
\hline Echantillon & Site & Matière & Profondeur $(\mathrm{cm})$ & Numéro & Age $14 \mathrm{C}$ & Déviation & cal. prob. 95\% & $\%$ & Remarques \\
\hline FLEURY $1 \mathrm{R}$ & SAA4 & Croute tesson & $x$ & $\begin{array}{c}\text { POZ- } \\
072622\end{array}$ & 65 & 30 & 1811-1921 AD & 71 & Out of range \\
\hline TAA 10 & SAA4 & Charbon & 60 & $\begin{array}{l}\text { POZ- } \\
109440\end{array}$ & 755 & 30 & $1215-1282 \mathrm{AD}$ & 95 & \\
\hline TAA 12 & SAA5 & Charbon & 45 & $\begin{array}{c}\text { POZ- } \\
109441\end{array}$ & 1830 & 30 & $117-252 \mathrm{AD}$ & 91 & \\
\hline TAA 17 & SAAG & Charbon & 35 & $\begin{array}{l}\mathrm{POZ}- \\
109442\end{array}$ & 1810 & 120 & $54 \mathrm{BC}-437 \mathrm{AD}$ & 92 & Petite quantité \\
\hline TAA 25 & SAA7 & Charbon & 60 & $\begin{array}{l}\mathrm{POZ}- \\
109326\end{array}$ & 1215 & 30 & 763-889 AD & 79 & \\
\hline TAA 31 & SAAB & Charbon & 70 & $\begin{array}{l}\text { POZ- } \\
109327\end{array}$ & 3985 & 35 & $2581-2453 \mathrm{BC}$ & 92 & \\
\hline $\begin{array}{l}\text { TAA } \\
\text { SAA }\end{array}$ & $\begin{array}{l}\text { Tarière an } \\
\text { Site arche }\end{array}$ & $\begin{array}{l}\text { éologique Alama } \\
\text { ogique Alama }\end{array}$ & $\begin{array}{l}\mathrm{OxCa} \\
\text { IntCa }\end{array}$ & $\begin{array}{l}\text { I v4.2.3 Br } \\
13 \text { atmosp }\end{array}$ & $\begin{array}{l}\text { Ramsey } \\
\text { ic curve }\end{array}$ & $\begin{array}{l}13) ; ~ r: 5 \\
\text { imer et al }\end{array}$ & 013) & & \\
\hline
\end{tabular}

- SAA 5 : Site à fossé

Ce site se trouve au sud dans le « coude » de la crique Alama sur le layon A. Il s'agit d'un beau plateau ayant livré une quinzaine de tessons ainsi qu'un niveau sombre et enfoui. Vers le nord, la pente est marquée par un fossé ressemblant à un chemin creux de quatre mètres de large qui descend depuis un replat vers la crique Alama. Son trajet linéaire de 230 mètres de long semble marquer l'accès à la crique et à la cascade, où des polissoirs ont été notés. La colline située symétriquement à celle-ci par rapport à la crique Alama n'a pas livré de tessons de céramique.

- SAA 6 : Site à fossé

Ce site se trouve sur le sommet d'une grande colline située entre le sommet en cloche et la crique Alama. Le sommet a livré une bonne quantité de tessons céramique ( $\mathrm{n}=67)$ ainsi qu'un niveau sombre enfoui. Vers l'est, qui descend vers un petit affluent de la crique Alama, la pente est marquée par un fossé de quatre mètres de large qui, depuis un replat, descend de façon linéaire sur 230 mètres vers la crique, comme celui du précédent site SAA 5.

- SAA 7 : Ancien village Akulio?

Ce site se trouve sur le sommet d'une grande colline située entre deux talwegs au sud de la crique Alama sur le layon A. Le sommet, envahi par une cambrouse récente et de jeunes arbres pionniers, a livré une bonne quantité de tessons de céramique $(n=44)$ et de charbons à faible profondeur, ainsi qu'un niveau sombre enfoui. Selon les Wayana il s'agit d'un site assez récent, peut-être des dits «Indiens sauvages » appelés Akulio par les Wayana, ou Oyarikulé dans la littérature ancienne.

- SAA 8 : Montagne couronnée

Ce site se trouve sur le sommet d'une crête dont la partie sommitale est fossoyée : il s'agit d'une montagne couronnée. Sur la crête le fossé est mieux visible que dans les pentes. En faisant le tour au GPS, l'enclos fossoyé semble avoir une forme elliptique. Il a une profondeur d'environ 2 mètres et une largeur de 6 mètres. Vers l'ouest se trouve un passage dans le fossé, soit un comblement du fossé qui représente une sorte de pont. $\mathrm{Au}$ milieu de l'enclos, qui mesure 180 mètres de longueur, se trouve une cambrouse avec un niveau sombre enfoui peu marqué. Quelques tessons de céramique $(\mathrm{n}=5)$ ont été trouvés en surface. Les Wayana n'avaient jamais vu un tel site ${ }^{3}$. 


\section{Les indices archéologiques Alama (IAA)}

21 - IAA 1 Un seul tesson a été trouvé sur cette crête qui se trouve en bordure de la crique Alama et qui mène vers le « Sommet en cloche ». Il peut s'agir d'un site archéologique... (Figure 7)

- IAA 2 Trois tessons ont été trouvés au pied du sommet en cloche en bas d'un petit éperon aux sources d'une vallée.

- IAA 3 Un seul tesson a été trouvé en pente de la crête qui longe le mont « Tukusipan ${ }^{4}$ ».

Figure 7 : Un tesson trouvé sur une motte de terre

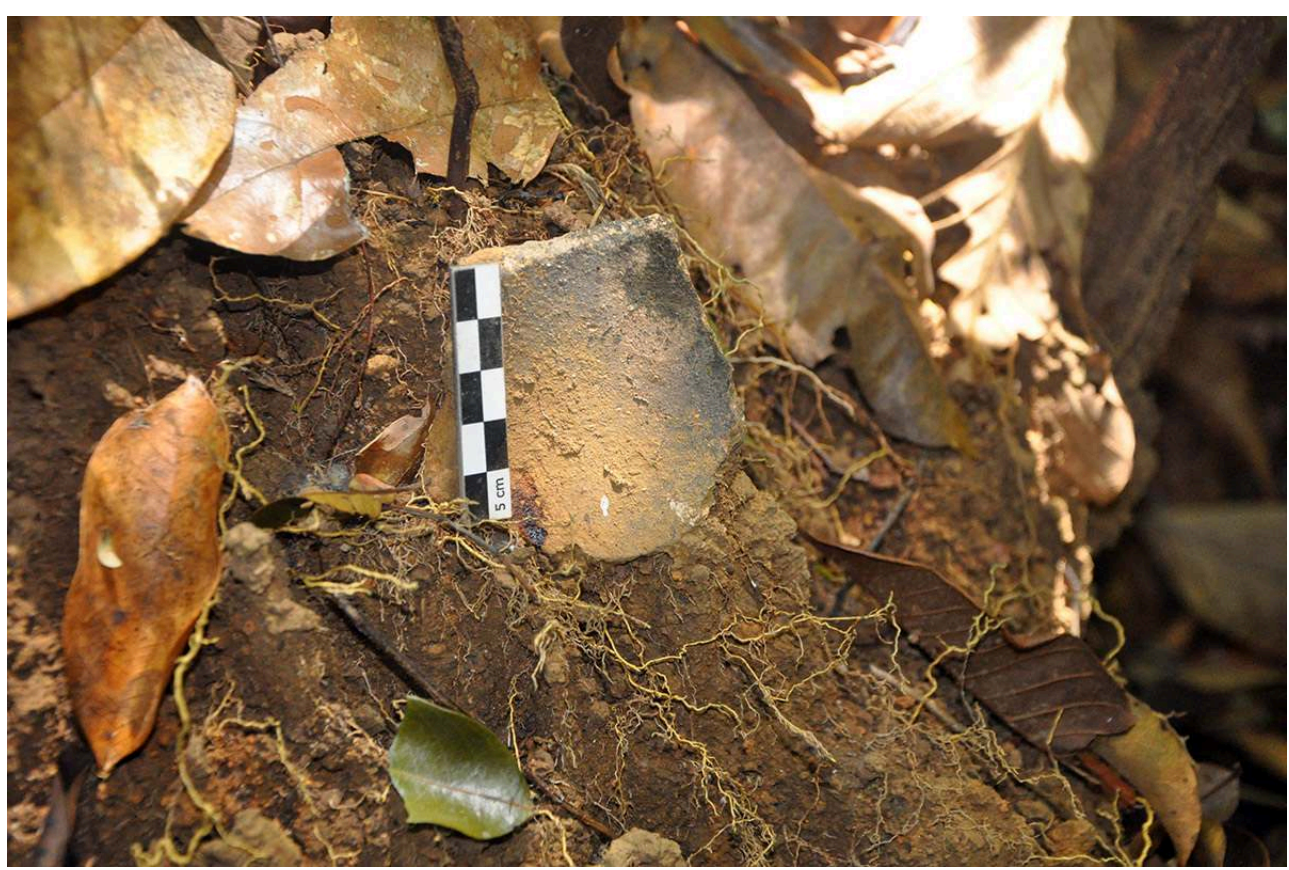

Cliché M. Fleury

\section{Discussion}

On remarque que la première datation (Fleury $1 \mathrm{R}$ ), qui est celle de la céramique trouvée en 2015 (site 4), représente la seule datation historique (Figure 6). Elle se trouve cependant " hors fourchette " car à partir du XvII e siècle notre atmosphère est polluée ce qui rend le résultat peu fiable. Cette céramique sert désormais d'indice et l'on retient seulement qu'il s'agit d'une datation attribuable à l'époque historique. L'autre date pour le site 4 , très fiable à deux sigma, nous montre une date précolombienne, se situant au début du deuxième millénaire de notre ère.

Concernant le résultat de la datation de la montagne couronnée (SAA8), il est étrange et concerne peut-être un fragment de charbon issu d'un paléo-feu (sécheresse) mais ne remet pas en cause une possible présence humaine il y a plus de 4000 ans... (Tardy 1998). Les dates les plus anciennes pour les montagnes couronnées en Guyane remontent au début de notre ère et perdurent jusqu'à avant la période de la découverte pour les différents types de sites fossoyés. 

roches herbeuses, cambrousses à graminées bambusiformes, formations à Phenakospermum guyannense, pinotières à Euterpe oleracea etc.) sont extrêmement variées. Très certainement la diversité des sols et de la topographie explique une part de cette diversité des paysages végétaux (Guitet et al. 2016), mais probablement aussi que les occupations humaines anciennes sont à l'origine d'une part importante de l'hétérogénéité observée du fait des perturbations qu'elles engendrent (Guitet et al. 2018). Cependant, à l'exception peut-être du site SAA7 dont le facies "forêt secondaire » paraît évident, le lien direct entre l'aspect de la végétation et l'histoire humaine est difficile à certifier formellement. Toutefois, l'hypothèse d'une empreinte durable laissée par l'agriculture sur les communautés végétales reste une hypothèse forte pour expliquer l'abondance des secteurs à forte densité de lianes, de Phenakospermum et de graminées bambusiformes. Bien que l'objectif de l'étude présentée ici ne soit pas d'en faire la démonstration, nous avons remarqué que la forêt de terre ferme peut être rapidement caractérisée par l'abondance du palmier Astrocarium sciophilum, qui occupe plus ou moins densément la strate inférieure. Ce palmier à croissance lente forme par place de belles palmeraies de sous-bois aux stipes hauts de cinq à six mètres; en d'autres secteurs aucun des palmiers ne présente un stipe dépassant un mètre de hauteur ; ces derniers sont donc manifestement beaucoup plus jeunes. Ailleurs, ce palmier est quasiment absent, les rares individus rencontrés ne présentent alors aucun stipe. Il se pourrait donc que ces secteurs à palmiers jeunes soient en phase de reconstitution après perturbation...

Malgré cette richesse des sites inventoriés et de l'apport de l'écologie, l'intérêt de cette mission ne réside pas dans le seul inventaire archéologique de la région. Notre travail pluridisciplinaire nous a permis de souvent confronter la vision des Wayana avec celle des scientifiques.

Le plus bel exemple s'est déroulé sur le troisième site (SAA 3) : sitôt arrivés sur le site, les Amérindiens ont remarqué "C'est un cimetière!». La présence de plusieurs excavations de forme allongée, leur a en effet rappelé leurs cimetières où les hommes sont enterrés à même le sol, avec une simple planche posée au-dessus. Lorsque celle-ci pourrit le sol s'effondre, laissant une dépression sur le sol (Figure 8). En l'absence de preuve formelle, l'écologue propose le déracinement simultané d'un grand nombre d'arbres comme hypothèse alternative expliquant ces excavations. Il relève toutefois que de manière assez inhabituelle, un dense mat racinaire tapisse le fond des dépressions. De plus, la fréquence sur le site (au moins une dizaine) et la forme évoquant un corps allongé, nous fait pencher pour l'hypothèse wayana, d'autant plus que la découverte, à proximité, de nombreuses céramiques prouve une occupation humaine assez importante. 
Figure 8 : Une tombe selon les Wayana

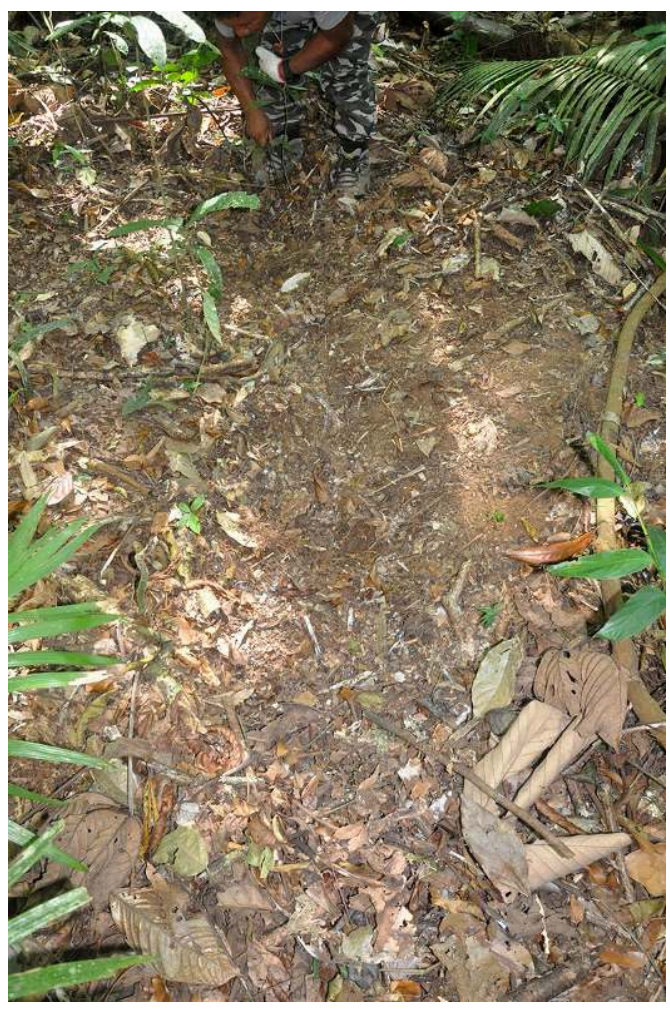

Cliché M. Fleury

28 La présence de ces tombes ne contredit pas la possibilité de la présence d'un ancien habitat, car « autrefois les morts étaient souvent enterrés dans le village, parfois même sous les carbets ", nous précisent les Wayana. La présence de trop de morts dans le village qui incitait à déplacer le village, comme d'autres facteurs tels le manque de ressources, l'épuisement des sols ou des guerres intertribales.

Le site de la montagne couronnée (SAA 8) : Bien que les Wayana n'aient jamais vu de montagne couronnée, il nous fait part de leur interprétation : selon eux,

" ces lieux auraient pu être des lieux d'entraînement militaire, réservés aux hommes, les femmes et les enfants n'ayant pas le droit d'y pénétrer. C'était sûrement l'école des guerriers, seulement les garçons avaient le droit d'y aller » (Mimi Siku, le 9/10/18).

Cette interprétation est imprégnée de l'histoire orale des Wayana, se rapportant au «premier village » (sous-entendu sur la Litani), Kulumulipan «le lieu des bambous »:

«C'était un village peuplé uniquement d'hommes, de guerriers. Les femmes n'avaient pas le droit d'y accéder, encore moins si elles avaient leurs règles ou étaient enceintes. Les enfants non plus n'avaient pas le droit d'y résider. Les bambous avaient été plantés pour faire des flèches (pointes de flèches)...C'était un village de réunions, très important, où l'on donnait l'autorisation de se déplacer sur le fleuve. Ce pourrait être le fameux village appelé Etakpapïtpë (le partage), ou bien Ehenokïtpë (l'envoi) » (Pidima 2013 in Fleury et al. 2016).

31 Ce village est considéré comme le premier village à partir duquel sont partis les différents groupes ethniques pour peupler les rivières, chaque groupe se voyant attribuer une rivière. 

cette histoire Wayana (XVIII-XIX) ), comme le montre sa datation, mais il n'est pas impossible que certains lieux aient été réemployés comme base militaire aux Wayana en guerre.

Il en est de même pour les fossés linéaires ou chemins creux (SAA 5 et SAA 6) qui ont pu être réutilisés à cette période : on fait appel ici au témoignage de Tony et Patris, qui ont découvert pour la première fois les Indiens Wayana appelés à l'époque « Rocoyens » sur le haut Marouni en 1766. Ceux-ci sont alors installés sur la crique Amana (petite crique à l'est du Mont Amana), qui est un affluent du Marouini. Ils sont en lien avec les Indiens du rio Coulicary (Culicari), affluent du Jary (Jari). Lors de leur deuxième voyage en 1769, Tony passe trois semaines chez les Roucouyennes, et décrit leurs villages fortifiés et une organisation militaire ${ }^{6}$ :

« de leur poste avancé ${ }^{7}$ jusqu'au premier village, il y a environ quatre lieues ; mais il faut observer que ce chemin est fait avec bien plus de soins encore; il a huit ou neuf pieds de large ; il est droit et aligné, comme s'il avait été fait au cordeau, jusqu'à une demi-lieue du village ; ...il est fermé et palissadé avec des gaulettes,... » (Tony 1769 : 55).

La description de ce chemin, que certains auteurs ont parfois considéré comme pure affabulation, nous évoque à présent les différents sites à fossé (SAA 5 et SAA 6) avec des chemins profonds, longs et rectilignes qui semblaient relier les villages aux criques avoisinantes. Cette corrélation entre traces archéologiques, témoignages historiques et tradition orale wayana, souligne tout l'intérêt de travailler de manière pluridisciplinaire et participative. Bien évidemment, la nature et la datation de ces fossés méritent plus d'attention et davantage de recherches seront nécessaires.

La découverte du site archéologique SAA 7 qui semblait le plus récent (une trentaine d'années) a également été l'occasion de confronter et cette fois-ci de conforter les deux interprétations : les Wayana y ont vu le même type de forêt que lorsqu'ils abandonnent leur abattis et y retournent une trentaine d'années plus tard. (Figure 9). L'écologue a effectivement daté la forêt de régénération à approximativement la même époque. Lorsqu'on a découvert ce site, les Wayana ont de suite évoqué la présence des Indiens Akulio, qu'ils appellent les «Indiens sauvages » (au sens étymologique bien sûr, c'est à dire « les Indiens de la forêt »). 
Figure 9 : Photos évoquant l'emplacement d'un « village récent »
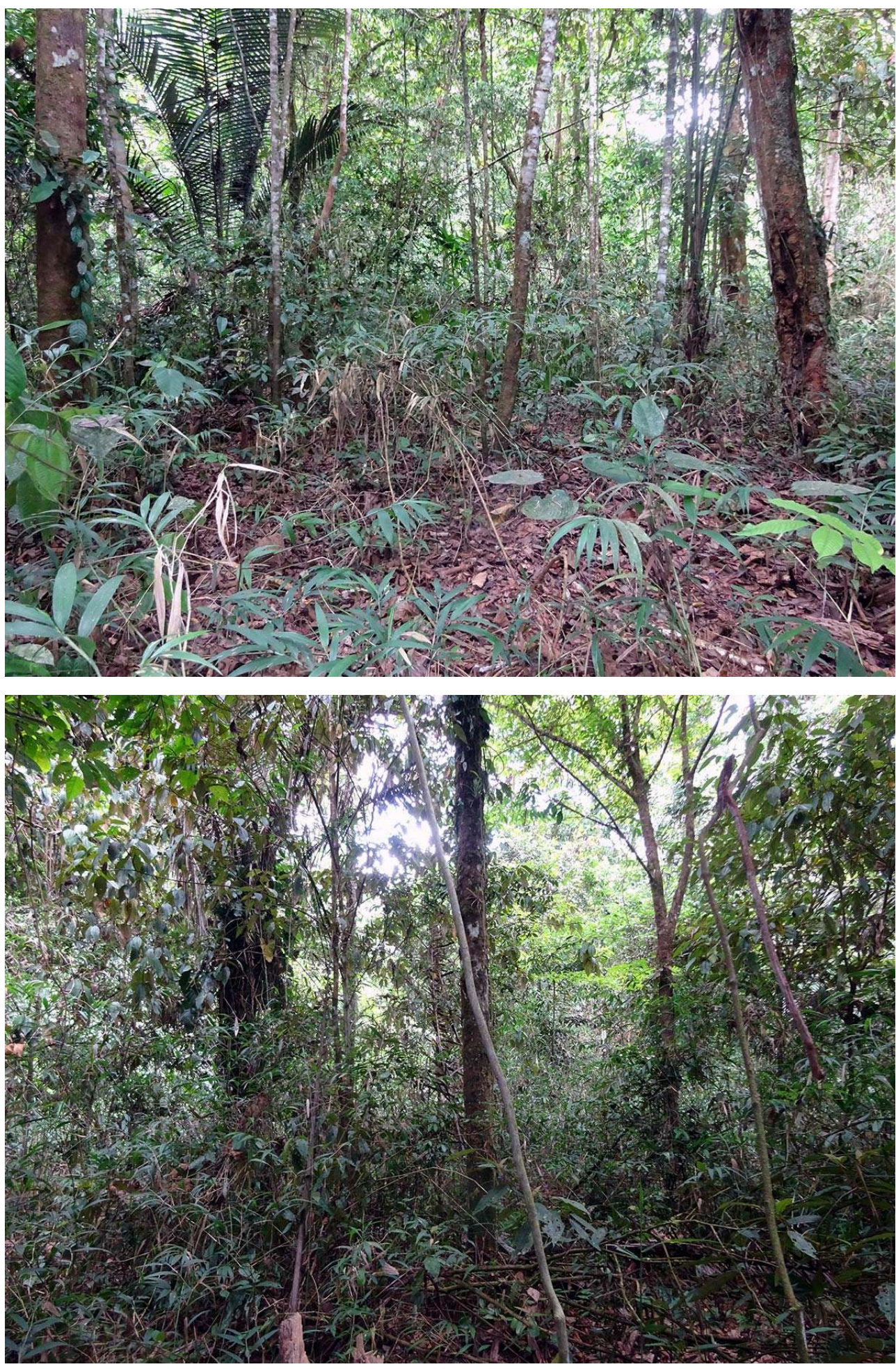

36 Le soir, Aimawalé nous conta l'histoire de son père, ancien militaire qui avait participé dans cette région, pour l'État du Suriname, à des razzias d'Indiens Akulio dans les années 1890 , afin de les ramener près des missions pour les évangéliser.

37 Bien sûr la découverte de l'emplacement de ce «village relativement récent » a donné lieu à de nombreuses discussions : s'il date des années 90, il est probable qu'il soit dû à la présence de ces Indiens nomades. D'autant plus qu'il n'y avait pas de trace d'abattis à 
proximité, et on n'a pas retrouvé de plantes cultivées : les Akulio ne cultivent pas, ce sont des chasseurs-cueilleurs (voir à ce sujet le témoignage de Deboer en 1970: Figures 10 et 11). De plus, l'absence de pécaris à lèvres blanches, seule viande prisée par les Akulio selon les Wayana, lors de nos prospections dans cette région évoquait certainement leur empreinte...

Figure 10 : Carte extraite de De Boer (1970) avec l'emplacement des tribus (Wamas (Wama) et Akurios (Akulio)) contactées et les dates correspondantes

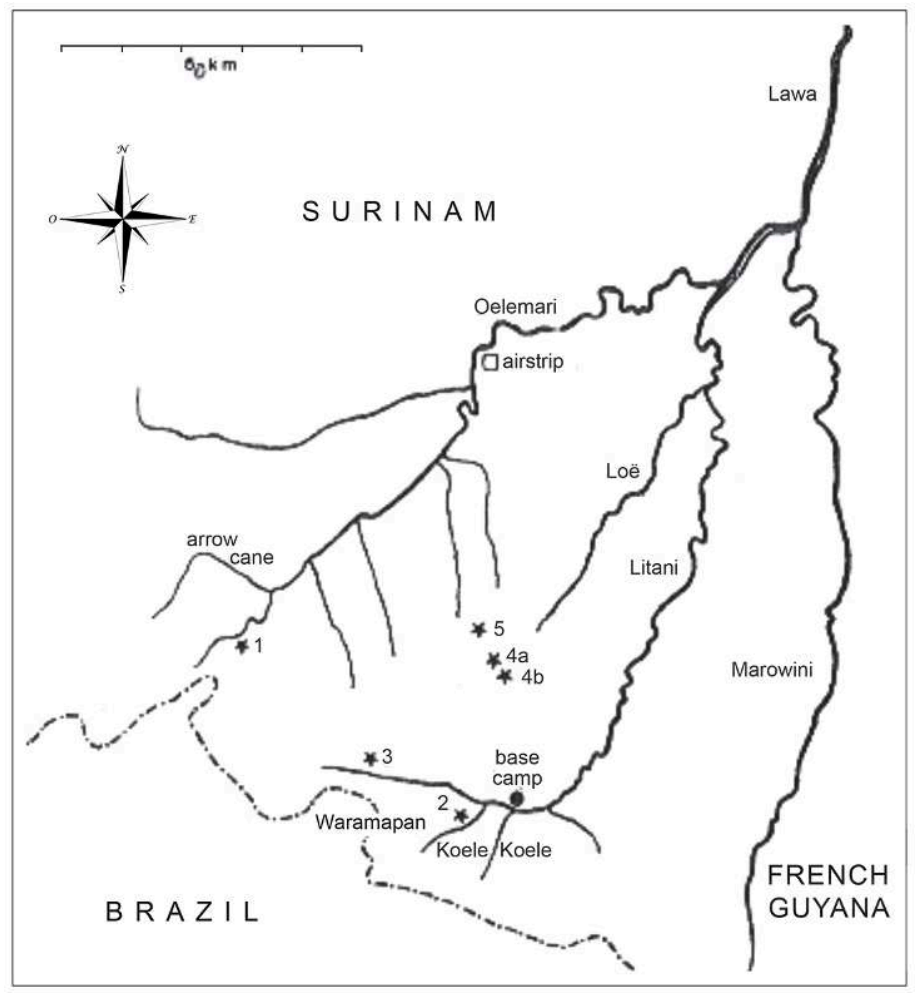


Figure 11 : Photo extraite du livre de De Boer (1970) avec une légende indiquant que les Indiens Akurios (Akulio) sont nomades (chasseurs-cueilleurs)

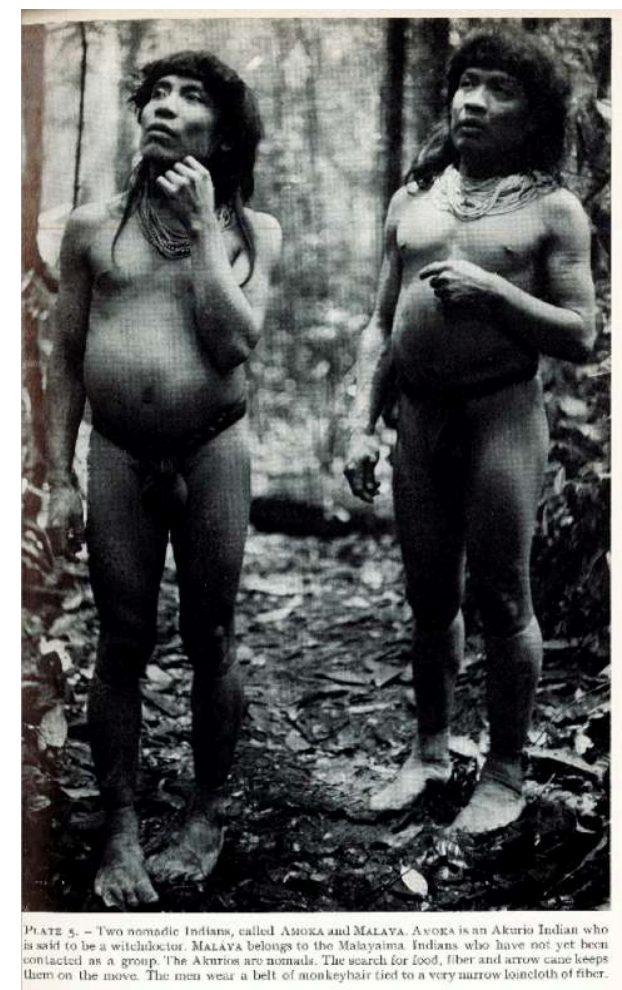

\section{Conclusion}

Les résultats de cette première prospection ont dépassé toutes nos attentes: non seulement nous avons pu mettre en évidence de nombreux sites archéologiques et documenter ceux qui étaient déjà connus, mais nous avons trouvé pour la première fois en Guyane, des traces de chemins linéaires de 4 mètres de large sur 230 mètres de long. Ces chemins se dirigeant vers la crique représentent la trace, selon l'archéologue, soit d'un passage intense pendant une courte période, soit d'un passage de quelques personnes sur une très longue période. Selon les datations (provisoires, en attente de confirmation par d'autres échantillons) ces sites dateraient du premier millénaire. Mais il est tout à fait envisageable que tous ces sites archéologiques aient été réoccupés pendant la période proto-Wayana, comme le laisse supposer la céramique trouvée à proximité de l'éperon barré. On peut même envisager que les proto-Wayana en guerre aient utilisé ces chemins déjà creusés pour y mettre une palissade et protéger ainsi les femmes qui allaient chercher de l'eau ${ }^{8}$ à la rivière (le rapt des femmes étant une cause très fréquente de guerre). Le témoignage de Tony, qui a séjourné dans une zone très proche (crique Amana), et décrivant des chemins droits, alignés, fermés et palissadés avec des gaulettes, corrobore cette hypothèse.

Cette expérience de recherche participative et pluridisciplinaire a été non seulement riche sur le plan scientifique mais aussi une très belle expérience humaine. Les Wayana ont pu participer à la recherche tout en se réappropriant une partie de leur histoire. Les soirées ont été également l'occasion d'échanges fructueux : les histoires d'Indiens «sauvages ", l'évolution du mode de vie des Wayana depuis cette période historique 
dans les Tumuc-Humac et le fait qu'ils ne souhaiteraient pas revenir en arrière. Ce mode de vie en forêt leur paraît dur et austère à présent qu'ils sont habitués à vivre au bord de la rivière avec le «confort moderne" (eau potable dans les villages, et électricité en cours d'installation). Ces soirées étaient aussi agrémentées par la lecture de récits historiques comme les témoignages de Coudreau, l'enregistrement de mythes et de récits wayana concernant cette époque, ou des expériences de vie des participants.

Ce travail a été restitué fin 2018 dans les écoles wayana d'Antécuma pata et de Taluwen, avec la participation des Wayana qui ont contribué au projet, et à la mairie de Maripasoula pour la fête de la science. Des documents vidéos et photographiques ont été réalisés en ce sens pour animer ces restitutions et en tirer le maximum d'intérêt pour les élèves et le grand public. Une publication ultérieure réunissant tous les travaux sur les anciens lieux de vie wayana est également prévue, comme support pédagogique à l'enseignement, de manière à ce que les jeunes Wayana puissent se réapproprier leur propre histoire.

Enfin, nous souhaitons poursuivre ces recherches en archéologie participative, sur la criquz Alama, si les financements le permettent, avec la participation de jeunes Wayana afin de mieux connaître et valoriser l'histoire de cette région, qui est loin d'avoir révélé tous ses secrets.

Cette mission a pu être réalisée grâce aux financements du Labex CEBA (programme REKABIOS), du Ministère des Outre-mer, du Parc Amazonien de Guyane (PAG) de l'UMR 208 PALOC MNHNIRD, de la Direction des Affaires Culturelles en Guyane (DAC - SRA), et la participation des agents de l'INRAP, de l'IRD, du MNHN et du CNRS-Guyane, que nous tenons tous à remercier. Ce travail a bénéficié du soutien des «Investissements d'avenir » de l'Agence nationale de la recherche française (Ceba, réf. ANR-10-LABX-25-01)

\section{BIBLIOGRAPHIE}

Abonnenc E. 1952 - Inventaire et distribution des sites archéologiques en Guyane française. Journal de la Société des Américanistes NS 41 : 43-62.

Ahlbrinck w. 1956 - Op zoek naar de Indianen. Verslag van een Expeditie naar de Zuidgrens van Suriname ter Opsporing en Bestudering van twee onbekende Indianenstammen: De Wama's en de Wajanikoele's. Mededeling CXVIII, Afdeling Culturele en Physische anthropologie 52. Amsterdam, Koninklijk Instituut voor de Tropen.

chapuis J. 2007 - L'ultime fleur. Ekulunpi tihmelë. Essai d'ethnosociogénèse wayana. Encyclopédie wayana. Orléans, Presses Universitaires Orléans.

Coudreau H. 1893 - Chez nos indiens : quatre années dans la Guyane française (1887-1891). Paris, Hachette. crevaux J. 1883 - Voyages dans l'Amérique du Sud. Paris, Hachette.

Deboer M.W.H. 1970 - Report of a Contact with the Stone-Age Indians in Southern Suriname. Nieuwe West-Indische Gids 47 (3) : 249-259. 
Duin R. 2004 - Ethno-archaeological reconnaissance in the Wayana homeland. Report of the Kailawa expedition in southwestern French Guyana, October 17-November 10, 2004, ms

Duin R. 2009 - Wayana Socio-political Landscapes: Multi-scalar Regionality and Temporality in Guiana. Thèse de Doctorat, Université de Floride, ms

Dupuy F. (Ed.) 2012 - Arpenteurs du Confin. Explorateurs de l'intérieur de la Guyane (1720-1860). Paris, La librairie des cultures 4 , CTHS.

Fleury M., Alupki T., opoya A. \& Aloïké w. 2016 - Les Wayana de Guyane française sur les traces de leur histoire. Cartographie participative sur le Litani (Aletani) et mémoire orale. Revue d'ethnoécologie 9. [En ligne :] http://ethnoecologie.revues.org/2711

Fleury M. 2018 - Gaan Mawina (Marouini) : Éléments pour une cartographie de l'histoire des Noirs marrons Boni/Aluku et des Amérindiens Wayana sur le haut Maroni, Revue d'ethnoécologie 13. [En ligne :] http://journals.openedition.org/ethnoecologie/3534

Guitet S., sabatier D., Brunaux 0., couteron P., Denis T., Freycon V., Gonzalez S., Hérault B., Jaouen G., Molino J.-F., Pélissier R., Richard-Hansen C. \& vincent G. 2018 - Disturbance Regimes Drive The Diversity of Regional Floristic Pools Across Guianan Rainforest Landscapes. Scientific reports $8: 3872$. [En ligne :] https://doi.org/doi:10.1038/\$41598-018-22209-9

Guitet S., Freycon V., Bruneaux O., Pélissier R., sabatier D. \& Couteron P. 2016 - Geomorphic control of rain-forest floristic composition in French Guiana: more than a soil filtering effect? Journal of Tropical Ecology 32 (1) : 22-40.

Goeje C.H. de 1905 - Van Papadron naar Majoli e.o. Tijdschrift van het Koninklijk Nederlands Aardrijkskundig Genootschap 22 : 931-986.

Goeje C.H. de 1908 - Verslag der Toemoek-Hoemak expeditie. Tijdschrift van het Koninklijk Nederlands Aardrijkskundig Genootschap 25 : 943-1169.

Grenand P. 1982 - Ainsi parlaient nos ancêtres : essai d'ethnohistoire wayãpi. Paris, oRSTOM, 420 p. (Travaux et Documents ; 148).

Hurault J., FRENAY P. \& ROUX Y. 1963 - Pétroglyphes et assemblages de pierres dans le sud-est de la Guyane française. Journal de la Société des Américanistes 52 :157-166

Mazière M. \& Mazière G. 1993 - Maripasoula, Montagne couronnée de Yaou. Bilan Scientifique de la Région Guyane $1992: 21-23$.

Mazière M. \& Mazière G. 1997 - La recherche archéologique en Guyane. In : Mazière G. \& Mazière M. (Ed.) L'archéologie en Guyane. Cayenne, APPAAG : 25-54.

Mestre M., Hildebrand M. \& Delpech s. 2013 - Yaou. Rapport final d'opération INRAP, ms

Migeon G. 2008 - Étude par thermoluminescence de six tessons en provenance du site de l'Abri Daniel, Borne 1, commune de Maripa-soula, Guyane (laboratoire Archéolabs). In : Saint-Jean D. \& Pellet E. (Ed.) Explorateurs d'Amazonie : Aventuriers de la Science en Guyane. Cayenne, Ibis Rouge.

Pellet E., saint-Jean D. (Ed.) 2006 - Au cœur des Tumuc Humac. Matoury, Ibis Rouge.

Petitjean Roget H. 1991 - 50 sites de montagne en Guyane française : contribution à l'inventaire archéologique d'Émile Abonnenc. In : L. Sickler Robinson (Ed.) Proceedings of the Twelfth Congress of the International Association of Caribbean Archaeology, Cayenne 1987. Martinique, International Association Caribbean Archaeology : 241-258. 
schmidt L. 1942 - Verslag van drie Reizen naar de Bovenlandse Indianenbewerkt door Gerold Stahel, Bulletin 58 DepartementLandbouwproefstation in Suriname.

Tardy c. 1998 - Paléoincendies naturels, feux anthropiques et environnements forestiers de Guyane française du Tardiglaciaire à l'Holocène récent. Approches chronologique et anthracologique. Thèse de Doctorat, Université de Montpellier II, ms

Tony C. 1769 - Voyage dans l'intérieur du continent de la Guyane, chez les Indiens Roucoyens par Claude Tony, mulâtre libre d'Approuague. In : Dupuy F. (Ed.) Les Arpenteurs des confins. Explorateurs de l'intérieur de la Guyane (1720-1860). Paris, CTHS : 49-93. (La librairie des cultures ; 4).

Vidal G. 2012 - Voyage d'exploration dans le haut Maroni (Guyanes française et hollandaise)

(1861). In : Dupuy F. (Ed.) Les Arpenteurs des confins. Explorateurs de l'intérieur de la Guyane

(1720-1860). Paris, CTHS : 231-281. (La librairie des cultures ; 4).

white c. 2010 - Kumako : a place of convergence for Maroonsand Amerindians in Surinam (SA).

Antiquity 84 : 467-479.

\section{NOTES}

1. Cette mission naturaliste qui a duré un peu plus d'un mois a permis à ce jour d'identifier une centaine de nouvelles espèces biologiques pour les Guyanes dont environ 70 nouvelles espèces pour la science.

2. Une mission de repérage sur l'Alama en novembre 2020, nous a permis de constater que les Mélipones sont toujours présentes sur la crique et de confirmer cette hypothèse.

3. Depuis l'inventaire archéologique d'Émile Abonnenc (1952: 52), les sites fossoyés circulaires sur les sommets de collines et plateaux sont appelés montagne couronnée en suivant la tradition orale saramacca. Ces derniers, lors de leurs fuites des plantations sur la rivière Suriname, se sont réfugiés sur un site fossoyé sur la haute Saramacca (cf. White 2010). La tradition orale des Wayãpi conçoit ces sites comme le travail des Kalana, un groupe aujourd'hui éteint (Grenand 1982 : 270).

4. Le mont Tukusipan est un inselberg à facies très particulier qui évoque une gueule de singe monstrueux figé. Il nous paraît très étonnant, ainsi qu'aux Wayana, qu'un tel nom lui soit attribué. D'autant plus que l'inselberg, peu éloigné à vol d'oiseau, que nous avons dénommé "Sommet en cloche » pendant la mission Planète revisitée ressemble beaucoup à un Tukusipan, carbet communautaire chez les Wayana, en forme de dôme... Une erreur de dénomination cartographique serait-elle à l'origine d'une telle confusion? C'est une hypothèse qui reste à explorer...

5. Bien que d'autres récits situent ce village (Etakpatpïtpë), qui remonte à l'époque de Kailawa, dans les Tumuc-Humac.

6. C'est le seul témoignage qui nous reste de ce deuxième voyage, Patris ayant perdu toutes ses notes dans un naufrage. Mais c'est aussi la seule description de ce militarisme chez les Wayana?

7. Occupé uniquement par des hommes armés.

8. Selon Mimisiku (cctobre 2018) « les Oyaricoulés se promenaient avec des grandes jarres pour transporter de l'eau sur leur dos, dans des catouris (hottes). Il en a vu sur la Wamahpan dans les années 70. Tous les Amérindiens de cette région avaient les mêmes techniques de jarres, les Akulio, les Tyrios, les Wama.... » 


\section{RÉSUMÉS}

Cette mission d'archéologie participative dans le massif du Mitaraka, fait suite à un travail de cartographie participative qu'a entrepris Marie Fleury sur les anciens lieux de vie des Wayana en 2013, et à une expédition naturaliste "Planète revisitée » organisée par le Muséum national d'Histoire naturelle et l'ONG Pronatura, dans le même secteur en 2015. Différents éléments archéologiques trouvés lors de cette mission laissaient supposer une occupation humaine ancienne. Par ailleurs l'histoire orale des Wayana situe la genèse de leur peuple dans cette région des Tumuc-Humac. Une mission de six personnes, trois chercheurs et trois Wayana, s'est déroulée en octobre 2018, et a pu mettre en évidence huit sites et trois indices archéologiques, avec une moyenne d'un site par $\mathrm{km}^{2}$. La confrontation des interprétations entre Scientifiques et des Wayana a enrichi de manière significative les connaissances sur l'histoire de cette région. Quelques datations radiocarbones confirment qu'elle a été occupée depuis au moins deux milles ans.

This indigenous archaeological mission in the Mitaraka region, part of the Tumuc Humac Plateau, is the continuation of a previous project led by Marie Fleury concerning the indigenous cartography of ancient Wayana dwellings as well as the naturalistic expedition Planète revisitée, organized by the National Museum of Natural History and the NGO Pronatura. Many archaeological objects were discovered during both missions, suggesting an important ancient human occupation of this particular region. Moreover, the oral history of the Wayana pin-points the genesis of their people in this particular part of the Tumuc Humac Plateau. In October 2018, three researchers and three Wayana went on a mission there and were able to identify eight archaeological sites and three more possible sites with an average of one site per $\mathrm{km}^{2}$. The discussion and comparison of the interpretations of these sites between scientists and Wayana has significantly enriched the historical knowledge of this region. A few radiocarbon dates confirmed that this area has been occupied for at least two thousand years.

\section{INDEX}

Keywords : participatory archaeology, history, Wayana, Mitaraka, Tumuc-Humac

Thèmes : Wayana

Index géographique : Guyane française

Mots-clés : archéologie participative, histoire, Mitaraka, Tumuc-Humac

\section{AUTEURS}

\section{MARIE FLEURY}

Directrice de l'antenne du MNHN, UMR 208 PALOC, BP 9016597323 Cayenne. marie.fleury@mnhn.fr

\section{AIMAWALE OPOYA}

Village Taluwen, 97370 Maripasoula

\section{AITALEWA PALANAIWA}

Village Antécume pata, 97370 Maripasoula 


\section{ANAIMA PUTPU}

Village Antécume pata, 97370 Maripasoula

DANIEL SABATIER

Écologue, IRD, Bd de la Lironde, TA A-51/PS2, 34398 Montpellier MARTIJN VAN DEN BEL

Archéologue, INRAP, 842 Chemin Saint-Antoine, 97300 Cayenne 